\title{
COMPARISON OF GHRELIN HORMONE AMONG PREGNANT SUDANESE WOMEN
}

\author{
Mutasem Mahmoud Abdallah Ahmed ${ }^{1}$, Kamal Ahmed Abdelsalam², Mutaz Ibrahim Hassan ${ }^{3}$
}

1Department of Clinical Chemistry, Faculty of Medical Laboratory Sciences, Shendi University, Khartoum, Sudan.

2Department of Clinical Laboratory Science, Applied Medical College, Shaqra University, Saudi Arabia.

${ }_{3}^{3}$ Department of Clinical Chemistry, Faculty of Medical Laboratory Sciences, Shendi University, Khartoum, Sudan.

\section{BACKGROUND}

\section{ABSTRACT}

Multipara is basically understood to be a parity of two or higher and has stable relationship with obstetric complaints. It could be seen as a reason for unhealthy maternal and neonatal outcome.

Aims and Objectives- 1 . to compare the levels of ghrelin hormone in multiparity with nulliparity and primi parity, 2. to assess the levels of ghrelin hormone in multiparity according to parity, age, gestational age, body mass index, number of miscarriages and type of delivery.

\section{MATERIALS AND METHODS}

This case control study of four hundred women aged 20-48 years from two hospitals in Khartoum state. Women were grouped into three groups comprising multiparity, primiparity and nulliparity. Questionnaires were given to the women and their blood samples were collected via vein puncture in early morning (8:00 - 11:00 am); after centrifugation of blood sample, these were analysed for ghrelin levels by using fully-automated ELISA. The sample size was taken for convenience.

\section{RESULTS}

Ghrelin levels showed significant changes in multiparity group when compared to nulliparity and also primiparity ( $\mathrm{p}$ value $<0.05$ ). In multiparity group, ghrelin levels showed insignificant changes when classified according to age and BMI, ( $p$ value $>0.05$ ); but showed significant change when classified according to parity, type of delivery and number of miscarriage ( $p$ value $<0.05)$.

\section{CONCLUSION}

Clinically, multiparity produces a significant variation in ghrelin level in pregnant women.

\section{KEY WORDS}

Multiparity, Primiparity, Nulliparity, Ghrelin.

HOW TO CITE THIS ARTICLE: Ahmed MMA, Abdelsalam KA. Hassan MI. Comparison of ghrelin hormone among pregnant Sudanese women. J. Evolution Med. Dent. Sci. 2019;8(07):412-415, DOI: 10.14260/jemds/2019/91

\section{BACKGROUND}

In medical practice, the parity is the number of times a lady has become pregnant and so attained the pregnancies to gestational age.[1] Any woman having birth a couple of times is termed multipara; while grand multipara illustrates the occurrence birth five or higher [2]. Grand multi parity is assumed to be an individual reason behind raising unhealthy consequences for both of fetus and also mothers particularly diabetics, antepartum and postpartum bleeding, malpresentation, caesarean section, iron deficiency anaemia, and a high perinatal mortality rate.[3] Newer studies have confirmed that in the existence of suitable perinatal attention, grand multiparity will not regarded an obstetrical trouble condition.[4] The majority of the studies argued that grand multiparas are more likely to be of old age which might be the reasons for increased morbidity and mortality. Such factor is difficult to be removed clinically because women's age is the most important biological variable that influences the reproductive health issue [5].

'Financial or Other Competing Interest': None.

Submission 27-09-2018, Peer Review 01-02-2019,

Acceptance 08-02-2019, Published 18-02-2019.

Corresponding Author:

Mutasem Mahmoud Abdallah Ahmed,

Department of Clinical Chemistry,

Faculty of Medical Laboratory Sciences,

Shendi University, Sudan. Khartoum, Sudan.

E-mail: mutasemlab3@gmail.com

DOI: $10.14260 /$ jemds $/ 2019 / 91$

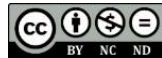

Few researches showed that maternal grand multiparity perhaps expects an additional complications of psychopathology in mature children as a third or later-born child have an increased risk of personality and behavioural disorders in adult life.[6] However, it remains unknown if the risks associated with maternal grand multiparity for mental disorders in the offspring persist after the transition into adulthood and to a more independent role in the society. It is also uncertain if maternal grand multiparity is independently associated with the risk of mental disorders or whether the associations found are confounded by maternal age at childbirth.[7]

Lenomorelin (INN),[8] the hunger hormone, is a neuropeptide hormone produced by ghrelinergic cells in the gastrointestinal tract. $[9,10]$ Besides regulating appetite, it also plays a significant role in regulating the energy.[11] When the stomach is empty, lenomorelin is secreted, but when the stomach is stretched, secretion stops. It works on brain tissue to escalate hunger level and also to raise gastric acid secretion accelerating gastrointestinal motility to organize the systems for coming nutrition [12]. On the other hand, it may decrease fertility [13]. Cord blood levels of active and total ghrelin show a correlation between lenomorelin levels and birth weight.[14] Within panic unrest, the nervous tension speeds up lenomorelin flow which proved to be important for emotional stress to upsurge scare response. So, it is regulated by stress even in the absence of adrenal hormones. Blocking the lenomorelin receptor during stress abolished fear stress without blunting other markers of stress. These results 
suggest that lenomorelin is a novel branch of the stress response. Human studies are needed to translate the use of anti- lenomorelin treatments to prevent stress-induced psychiatric disorders.[15]

And therefore, the aim of this study was to estimate effect of multiparity on ghrelin hormone compared to primiparity and nulliparity in Sudanese pregnant women.

\section{MATERIALS AND METHODS}

This study was designed to be a case control study, this study was conducted in Khartoum State, Jabal Awlia Hospital, and Omdurman Maternity Hospital. The study was carried out during the period from September 2016 to April 2019. Four hundred women were enrolled in this study; 200 were multiparity pregnant women as study group, 100 were primiparity pregnant women as control positive group and 100 were nulliparity (Non-pregnant women) were the control negative group. The sampling technique used was convenience sampling. All participants were in their fertility period and/or premenopausal period, in age of 20-48 years old. Women were grouped into three groups comprising multiparity, primiparity and nulliparity. Questionnaires were administered to the women and their blood samples were collected via vein puncture in early morning (8:00 - 11:00 am). After centrifugation of blood sample, these were analysed for ghrelin hormone levels by using full-automated ELISA. All pregnant women were at 26-34 weeks gestational age. Since the duration of the study was less. The women were selected by convenience sampling technique. The sample size required was taken for convenience.

\section{Ethical Considerations}

This case control study was approved by the research committee - College of Medical Laboratory Sciences-Shendi University. Informed consent was obtained from each participant before taking the samples.

Venous blood was collected using antiseptic for the skin, as well as data concerning any sample from clinical chemistry laboratories according to Inclusion criteria and exclusion criteria. Seven ml venous blood samples were obtained from each female using standard venipuncture technique, [16] in serum separator tubes (SST). After 15 minutes, serum specimens were collected in plane container after centrifugation at $3000 \mathrm{rpm}$ for 5 minutes. The serum stored frozen $\left(-20^{\circ} \mathrm{C}\right)$ in a tightly sealed tube for only 2 weeks and then analyzed. Specimens should be allowed to come to room temperature and then mixed thoroughly by gentle inversion before assaying. Then ghrelin was measured by automated Enzyme-Linked Immunosorbent Assay (ELISA) kit.

\section{Quality Controls and Managements}

Blood was collected with care and adequate safety precautions to ensure test results were reliable. Quality Assurance (QA) and standard Operating System was followed for all biological and clinical tests to achieve validity and reliability of test results.

\section{Methods of BMI Estimation}

It calculates a value indicative of the fat content of the body by dividing the weight by the square of height.[17]

$$
\begin{aligned}
& \mathrm{BMI}=\frac{\operatorname{mass}(\mathrm{kg})}{{\text { (height }(\mathrm{m}))^{2}}^{2}} \\
& \text { BMI Categories }
\end{aligned}
$$

\begin{tabular}{|c|c|}
\hline Categories & BMI \\
\hline Underweight & Less than 18.5 \\
\hline Normal Weight & $18.5-24.9$ \\
\hline Overweight & $25-29.9$ \\
\hline Obese & 30 or Higher \\
\hline
\end{tabular}

All pregnant women take drugs affecting on estimation and/or with major hormonal disorder along with those refused to participate in this study were excluded.

\section{Statistical Analysis}

All data are presented as mean \pm SD. Statistical significance was determined using one-way ANOVA followed by Tukey's multiple comparison test. In results with only two set of data, unpaired student's test with Welch correction (unequal sample size) was used. Though the SD values were high in some parameters, ANOVA was used as it has been reported to be robust for different types of data.[18]

\section{RESULTS}

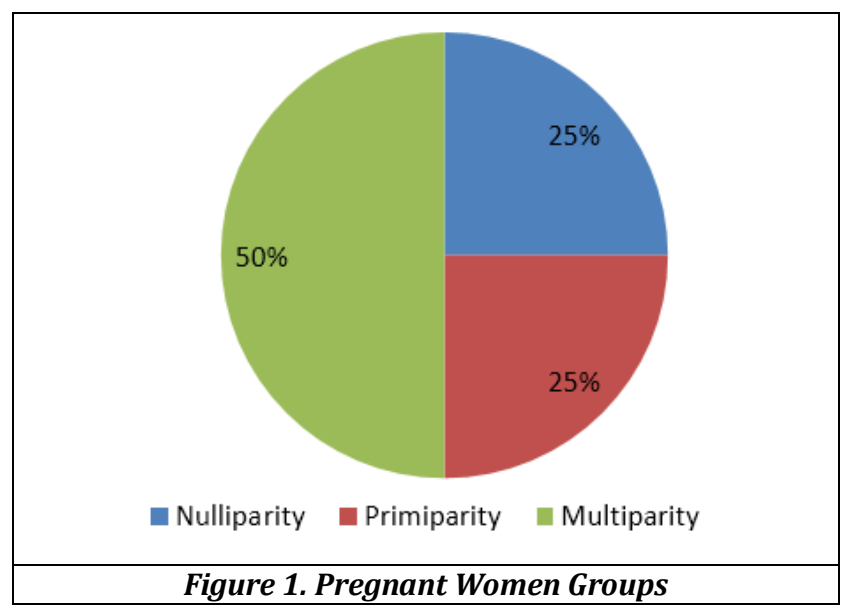

\begin{tabular}{|c|c|c|c|}
\hline Parameters & Nulliparity & Primiparity & Multiparity \\
\hline Ghrelin & $264.5 \pm 269.6$ & $789 \pm 708^{* * *}$ & $\begin{array}{c}565 \pm \\
1008.9^{* * *} \text { aaa }\end{array}$ \\
\hline BMI & $23.6 \pm 2.0$ & $30.6 \pm 24.5^{*}$ & $29.6 \pm 20.2^{*}$ \\
\hline Age & $32.1 \pm 2.7$ & $25.5 \pm 3.6^{* *}$ & $30.2 \pm 4.0^{* * *}$ aaa \\
\hline Table 1. Statistics of Demographic Data and Biochemical \\
Parameters
\end{tabular}

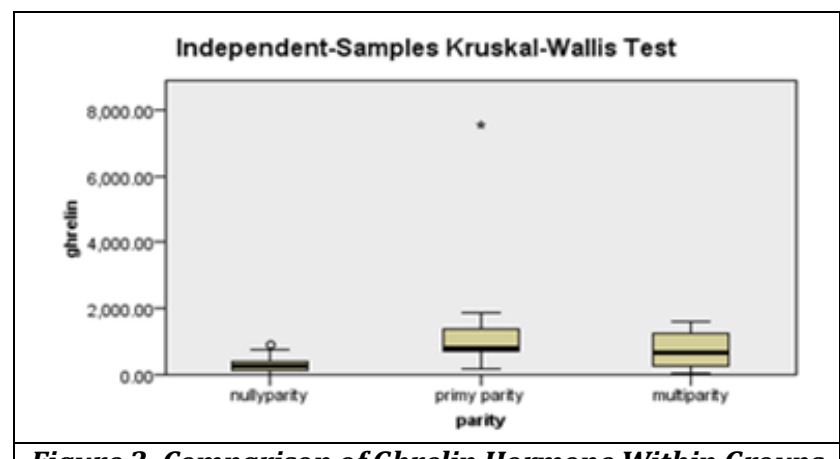

Figure 2. Comparison of Ghrelin Hormone Within Groups 
Table 1 and Figure 2: shows the statistics of demographic data and biochemical parameters computed for the three groups (Nulliparity, Primiparity and Multiparity). The results showed that there is statistical significance difference between the groups means for all parameters respectively.

\begin{tabular}{|c|c|c|c|c|c|c|}
\hline \multirow{2}{*}{ Parameters } & \multicolumn{2}{|c|}{ Multiparity } & \multicolumn{2}{c|}{ Primiparity } & \multicolumn{2}{c|}{ Nulliparity } \\
\cline { 2 - 7 } & $\mathbf{r}$ & $\begin{array}{c}\mathbf{p}- \\
\text { Value }\end{array}$ & $\mathbf{r}$ & $\begin{array}{c}\mathbf{p}- \\
\text { Value }\end{array}$ & $\mathbf{r}$ & $\begin{array}{c}\mathbf{p}- \\
\text { Value }\end{array}$ \\
\hline Age-Ghrelin & 0.314 & 0.000 & 0.120 & 0.233 & 0.006 & 0.953 \\
\hline BMI- Ghrelin & 0.029 & 0.683 & -0.050 & 0.625 & -0.045 & 0.0659 \\
\hline Table 2. Spearman Correlation Coefficient (R) Between \\
Demographic Data and Biochemical Parameters Within \\
Groups \\
\hline
\end{tabular}

Table 2. There was a week positive correlation between ghrelin and age among multiparous women. This correlation was found to be statistically significant.

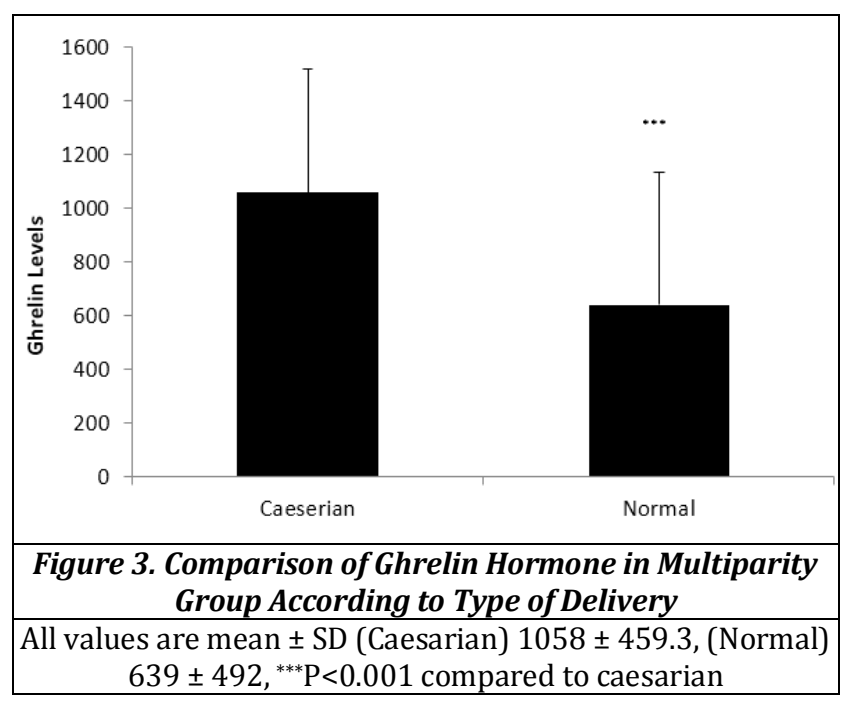

There were significant changes in ghrelin hormone according to type delivery, in which the highest value was in the pregnant women delivered by caesarean section and the lowest value where in the pregnant women delivered by normal term ( $\mathrm{p}$ value $=0.001$ )

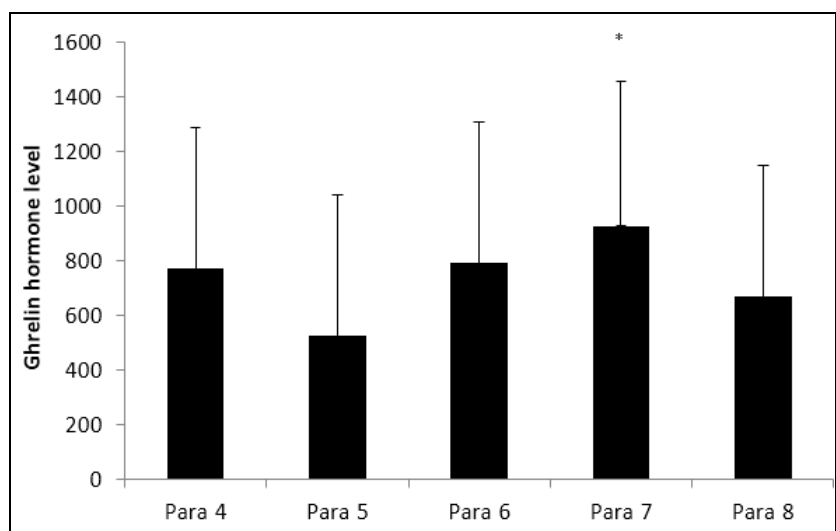

Figure 4. Comparison of Ghrelin Hormone in Multiparity Group According to Number of Parity

All values are mean $\pm S D$, (para 4), $769 \pm 518.4, n=71$, (para 5), $523 \pm 518.4, \mathrm{~N}=50$,

(para 6), 789.3 $\pm 516.5, \mathrm{n}=49$, (para 7), $927.41 \pm 530.2$, $\mathrm{n}=18$, (para 8), $668.1 \pm 479, \mathrm{n}=12 .{ }^{*} \mathrm{P}<0.05$ compared to Para 5
There were significant changes in ghrelin hormone according to number of parity, in which the maximum value were in para seven pregnant women and the minimum value where in para five pregnant women ( $\mathrm{p}$ value $=0.017$ )

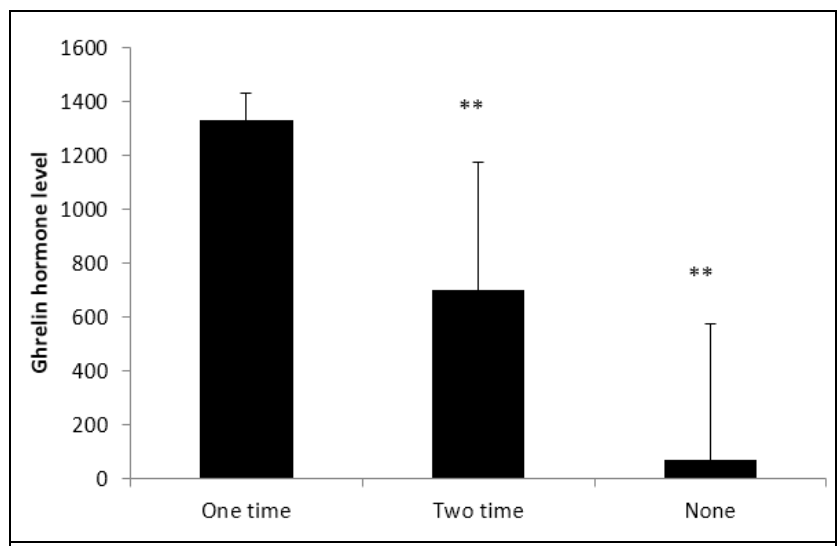

Figure 5. Comparison of Ghrelin Hormone in Multiparity Group According to Number of Miscarriages

All values are mean \pm SD, (one time), $1330.1 \pm 100.84, \mathrm{n}=12$,

(two time), $700 \pm 472, \mathrm{n}=5$ (none), $681 \pm 506, \mathrm{n}=183$.

${ }^{* *} \mathrm{P}<0.01$ compared to one time

There were significant changes in ghrelin hormone according to number of miscarriages, in which the highest value was in pregnant women has one time of miscarriage and the lowest value where in non-miscarriage pregnant women $(p$ value $=0.000)$.

\section{DISCUSSION}

The pregnancy is involved with multiple physiological and morphological modifications to fit the requirements of the growing fetus. Those variations are concerned to produce deleterious results for mother and infant due to the number of gestations.

In the current study, the results displayed that the mean level of ghrelin in pregnant females increases significantly (p. value $<0.05$ ) compared to non-pregnant (Nulliparity), but the results showed significant low levels of ghrelin in multi parity and significant high levels of ghrelin were in primiparity pregnant ladies. The same results were found in the report of Onur et al.[19] that also mentioned that preeclampsia can increase ghrelin significantly.

In this study, the ages of the multi parity pregnant women were divided into three groups, less than (or equal to) 29 years old ( $45 \%$ of participants), from 30-41 years old (54.25\% of participants) and more than (or equal to) 42 years old $(0.75 \%$ of participants); accordingly, the results of ghrelin hormone showed insignificant changes ( $\mathrm{p}$ value < 0.05). And proved to these results, the study of Victoria et al.[20] Concluded significant age-related changes in the female hormones during reproductive years.

The BMI in multiparty group within this study showed insignificant effect in the mean level of ghrelin hormone ( $p$ value $>0.05$ ); nevertheless, previous studies were showed that multi parity being a risk factor for obesity in later life either before or after menopause and definitely real association between the number of parities and obesity was noticed.[21]

The delivery type of multi parity women in this study showed significant alteration in the mean level of ghrelin 
hormone, in which the maximum value was in the pregnant women delivered by caesarean section, and the minimum value where in the pregnant women delivered by a normal term. This result is opposite to the report of Abel Hakeem et al.[22] who concluded that delivery mode will not affect the cord ghrelin levels.

In this study, the number of parity of multiparity pregnant women showed significant effect in the mean level of ghrelin hormone in which the maximum value was in $7^{\text {th }}$ parity and the minimum value was in $5^{\text {th }}$ parity. This result proved the report of Alan et al.[23] who suggesting a profound effect of parity on maternal hormones levels.

The number of miscarriages of pregnant women within this study displayed significant effect in the mean level of ghrelin hormone ( $\mathrm{p}$ value $<0.05$ ). The results exhibited that pregnant woman with one time of miscarriage has the maximum ghrelin value while the minimum value was in pregnant women with zero time of miscarriage.

\section{CONCLUSION}

The results suggest that the levels of ghrelin hormone was significantly affected by multi parity, delivery type, parity and number of miscarriages of the pregnant women.

\section{REFERENCES}

[1] Creinin MD, Simhan HN. Can we communicate gravidity and parity better? Obstet Gynecol 2009;113(3):709-11.

[2] Gary C. Williams Obstetrics. 22 ${ }^{\text {nd }}$ edn. McGraw-Hill Companies 2005: p. 121.

[3] Al JF. Grandmultiparity: a potential risk factor for adverse pregnancy outcomes. J Reprod Med 2012;57(1-2):53-7.

[4] Kumari AS, Badrinath P. Extreme grand multiparity: is it an obstetric risk factor? Eur J Obstet Gynecol Reprod Biol 2002;101(1):22-5.

[5] Alsammani MA, Ahmed SR. Grand multiparity: risk factors and outcome in a tertiary hospital: a comparative study. Mater Sociomed 2015;27(4):2447.

[6] Fazel S, Bakiyeva L, Cnattingius S, et al. Perinata risk factors in offenders with severe personality disorder: a population-based investigation. J Pers Disord 2012;26(5):737-50.

[7] Lahti M, Eriksson JG, Heinonen K, et al. Maternal grand multiparity and the risk of severe mental disorders in adult offspring. PLoS One 2014;9(12):e114679.

[8] Sakata I, Sakai T. Ghrelin cells in the gastrointestinal tract. International Journal of Peptides 2010;2010:945056.

[9] Inui A, Asakawa A, Bowers CY, et al. Ghrelin, appetite, and gastric motility: the emerging role of the stomach as an endocrine organ. FASEB Journal 2004;18(3):439-56.
[10] Dickson SL, Egecioglu E, Landgren S, et al. The role of the central ghrelin system in reward from food and chemical drugs. Molecular and Cellular Endocrinology 2011;340(1):80-7.

[11] Burger KS, Berner LA. A functional neuroimaging review of obesity, appetitive hormones and ingestive behavior. Physiology \& Behavior 2014;136:121-7.

[12] Schwartz MW, Woods SC, Porte D Jr, et al. Central nervous system control of food intake. Nature 2000;404(6778):661-71.

[13] Comninos AN, Jayasena CN, Dhillo WS. The relationship between gut and adipose hormones and reproduction. Human Reproduction Update 2014;20(2):153-74.

[14] Yokota I, Kitamura S, Hosoda H, et al. Concentration of the n-octanoylated active form of ghrelin in fetal and neonatal circulation. Endocrine Journal 2005;52(2):271-6.

[15] Meyer RM, Burgos-Robles A, Liu E, et al. A ghrelingrowth hormone axis drives stress-induced vulnerability to enhanced fear. Molecular Psychiatry 2014;19(12):1284-94.

[16] NCCLS: Procedure for the collection of diagnostic blood specimens by Venipuncture: Approved Standard, Sixth Edition. Wayne, PA: CLSI Document (H3-A6). Vol. 27. No. 26. 2007.

[17] Young S. Student views of effective online teaching in higher education. American Journal of Distance Education 2006;20(2):65-77.

[18] Blanca MJ, Alarcón R, Arnau J, et al. Non-normal data: Is ANOVA still a valid option? Psicothema 2017;29(4):552-7.

[19] Onur E, Hamit YE, Hulya A, et al. Increased serum ghrelin in preeclampsia: Is ghrelin a friend or a foe? Ginekologiapolska 2016;87(4):277-82.

[20] Victoria CM, Delwood CC, Paul IM, et al. Age-related changes in the female hormonal environment during reproductive life. American Journal of Obstetrics and Gynecology 1987;157(2):312-7.

[21] Hajiahmadi M, Shafi H, Delavar MA. Impact of parity on obesity: a cross-sectional study in Iranian women. Medical Principles Practice 2015;24(1):70-4.

[22] Hakeem AAH, Saed SZ, El Rehany MA, et al. Serum level of ghrelin in umbilical cord in small and appropriate for gestational age newborn infants and its relationship to anthropometric measures. J Clin Neonatal 2012;1(3):135-8.

[23] Alan AA, Anne ZJ, Annekatrin L, et al. Effects of parity on pregnancy hormonal profiles across ethnic groups with a diverse incidence of breast cancer. Cancer Epidemiol Biomarkers Prev 2006;15(11):2123-30. 